\title{
Performing Vaginal Prolapse Surgery as a Day Case Procedure
}

\author{
Asfour V* and Penny J \\ Clinical Fellow in Urogynaecology, St Mary's hospital, London, UK \\ Consultant in Obstetrics and Gynaecology, East Surrey hospital, UK
}

Submission: March 17, 2017 ; Published: May 02, 2017

*Corresponding author: Asfour V, Clinical Fellow in Urogynaecology, St Mary’s hospital, London, UK, Email: victoria.asfour@imperial.nhs.uk

Abstract

Introduction: In-hospital stay is known to be associated with higher cost and clinical risk.

Study design: All consecutive vaginal repairs performed under a single surgeon in a district general hospital between 2013-2015 were retrospectively analysed. Standard surgical technique under general anaesthesia was used for all cases. Primary outcome measures were inhospital stay and urinary retention leading to catheterisation. Post-operative retention of urine was managed with an indwelling urinary catheter for 2-3 days.

Results: Fifty-seven patients, median age 65yr (42-88yrs), underwent surgery. 56\% had a previous hysterectomy. 56\% (32/57) were performed as day case procedures. Median length of stay was 5 hours (2.5-11 hrs). 11 went home the next day. 10\% (6/57) went home with a catheter for $2-3$ days. $14 \%$ had vaginal packs.

Conclusion: In dwelling catheter and vaginal packs prolong hospital stay. Shorter hospital stay is preferred by patients.

Keywords: Prolapse; Thromboembolism; Anaesthesia; Catheterisation

\section{Introduction}

Vaginal wall prolapse is very common affecting up to a third of women. Surgical vaginal wall fascial repair is considered when conservative measures fail or are not acceptable to the patient.

Day case surgery offers clinical advantages of earlier mobilisation, reducing venous thromboembolism and hospitalacquired infections. Avoiding the need of in-patient beds, reduces to the cost of care, and allows to patient to have her surgery even if there are no in-patient beds available in the hospital, therefore avoiding cancellations. Day case surgery is preferred by patients as it allows return to their home environment and normal daily activities sooner.

We aimed to observe the feasibility of performing native tissue fascial vaginal wall prolapse surgery, excluding hysterectomy, as day case, and assessed the patient acceptability and satisfaction.

\section{Methods}

This study has 2 sections. First is the patient satisfaction questionnaire, and second is the retrospective review of records.

\section{Patient records review}

All consecutive vaginal prolapse repairs performed under a single surgeon between 2013-2015 were retrospectively analysed by reviewing patient records. We included patients having anterior colporrhaphy, posterior colporrhaphy, both anterior and posterior colporrhaphy, or vaginal sacrospinous fixation. Some patients also had concurrent transobturator tape as well at the same time. Patients having only a transobturaor tape alone were excluded. Patients that had a hysterectomy as part of the prolapse repair procedure were excluded. Patients that had a hysterectomy previously in their gynaecological history were included.

Standard general anaesthetic was used for all cases. Bupivacaine $0.25 \%$ with 1:200 000 Adrenaline was used for hydro-dissection and haemostasis. Anterior and posterior

colporrhaphy were per-formed in the standard way using Buttress sutures and purse string sutures. The same techniques were applied in both in-patients and day care patients.

Primary outcome measures were in-hospital stay and urinary retention leading to catheterisation. Post-operative retention of urine was defined as failure to void over $200 \mathrm{ml}$ urine, with a less than $100 \mathrm{ml}$ residual on two consecutive occasions. Urinary retention was managed with an indwelling urinary catheter for 2-3 days. 
Patients are given post-operative care information at the preoperative assessment and on dis-charge from hospital, including contact numbers for post-operative advise. Patients are followed up in the lead clinician's clinic 3 months post-operative to address any ongoing problems.

\section{Patient satisfaction survey}

Patient satisfaction questionnaire was posted to all patients who had vaginal surgery including va-ginal prolapse repairs (colporrhaphy), transobturator tapes, sacrospinous fixation and hysterectomy by the senior author as lead clinician, including both as in-patient or day case procedure patients. Patient names were identified using the hospital coding database and theatre logbooks. The questionnaires were returned to the clinical audit office.

The questions of the patient satisfaction questionnaire were selected from the hospital clinical go-vernance question bank. These questions are used in several NHS patient satisfaction surveys across many specialties. The questions were generated and validated by the Surrey and Sussex Healthcare Trust.

Results

\section{Patient records review results}

Fifty-seven patient records were reviewed. All patients had a vaginal prolapse repair. They had a median age of 65 years (4288yrs). 56\% (32/57) had a previous hysterectomy reported in the gynaecological history. 56\% (32/57) were performed as day case procedures.

Median length of stay was 5 hours $(2.5-11 \mathrm{hrs}) .97 \%$ of day cases stayed under 8 hours. 11 went home the next day. 3 patients stayed for 2 days. 11 not ascertained. Reasons for inpatient stay included: age, hypertension, social reasons and declining an indwelling catheter.

$10 \%(6 / 57)$ went home with a catheter for 2-3 days; of these 3 were day case procedures and 3 were in-patients. Vaginal packs were used for $14 \%$ of patients ( 5 overnight, 3 for $1-6$ hrs post-op only). 1 patient returned the next day for PV bleeding that settled spontaneously.

\section{Patient satisfaction survey results}

202 women who had prolapse surgery and trans-obturator tapes were surveyed between 2010 and 2015. 71/202 (35\%) responded. $37 / 71(52 \%)$ had a colporrhaphy \pm sacrospinous fixation and 23/71 (32\%) had a vaginal hysterectomy.

$16 / 71(23 \%)$ were day-case procedures. Of the day case procedures $14 / 16$ had colporrhaphy \pm sacrospinous fixation and $2 / 16$ had sacrospinous fixation alone. 14/16 (87\%) of day case patients felt ready to go home on the same day. All hysterectomy patients stayed overnight (in-patients with a urinary catheter). Other reasons for in-patient stay included medical complications, urinary retention, pain and social problems. 11/71 (15\%) of all patients reported post-operative urinary retention.

$61 / 71(85 \%)$ recalled having an information leaflet before the day of the surgery; $59 / 71$ (83\%) felt the information in the leaflet had just the correct content. Full understanding of the information was reported in $92 \%$ of cases. $91 \%$ felt that were treated with respect and dignity. There were no re-admissions for patients discharged the same day.

Vaginal repairs that were done as in-patients were for patients that required post-operative admission for their medical co-morbidities, lack of support at home post-operatively and frail elderly. Patients were also managed as inpatients when unexpected difficulties were encountered, such as difficult haemostasis intra-operatively. All transobturator tapes were performed as day case pro-cedures. All hysterectomies were performed as in-patients.

\section{Discussion}

The first case series of doing vaginal repairs as day case procedures was proposed by Miklos JP et al. [1] in 1995. They achieved this by using local anaesthetic techniques, avoiding a general anaes-thetic.

Day case prolapse surgery has been described in the context of using different anaesthesia or surgical techniques [1-4]. The Maid stone team have described vaginal prolapse repair (colporrha-phy) using local anaesthetic $(20 \mathrm{ml}$ Bupivacaine $0.25 \%$, with 1:200 000 Adrenaline) and sedation (Midazolam $2 \mathrm{mg}$ intravenously with Remifentanil), whilst the patient received $40 \%$ oxygen via a face-mask.1 Kuhn A et al (2006) described anterior and posterior wall repairs under local anaesthetic only, with $63-88 \%$ improvement on POP-Q scores at 30 months.3

It is common UK practice for patients to go home a few hours after a general anaesthetic after a wide array of other procedures such as transobturator tapes and operative laparoscopy. In consultation with the anaesthetic team, we do not think that there is any advantage in the use of sedation over general anaesthesia. There was no expectation of a delay in discharge as a result of the anaesthetic.

In our experience, where all the patients had a standard general anaesthesia, the main determinant of length of stay, and therefore being discharged as a day case, was the management of a vaginal pack and catheter. We propose that the management protocol of the vaginal pack and urinary catheter, is the determinant of length of stay rather than the type of anaesthesia employed. A study into the use of vaginal packs from Medway showed that patients undergoing vaginal prolapse repair only had minimal bleeding, and removal of the pack was associated with some discomfort [5]. Routine use of vaginal packs are of unproven therapeutic benefit. 
The design and size of this study are not sufficient to conclude any difference in complication rate or long-term outcome; as such a study would need to be prospective, randomised and adequately powered.

In a cross-sectional study of patient satisfaction in 2401 patients undergoing day case surgery found that good preoperative preparation is associated with better post-operative satisfaction [6]. We use the RCOG Recover Well leaflets in the preassessment package, giving the patient sufficient time to read, prepare and have the opportunity to ask questions regarding their recovery before the operation.

\section{Conclusion}

We found that day case vaginal repair surgery is safe, acceptable and preferable to patients. There must be adequate protocols in place for bladder care, out-of-hours access for advise and admission to the gynaecology ward in case of any problems requiring overnight stay if needed. There needs to be appropriate follow-up care for patients that need to be discharged with a catheter. Further research is required to ensure that the long term outcomes are as successful as the in-patient procedure.

\section{References}

1. Miklos JP, Sie EH, Karram MM (1995) Vaginal correction of pelvic organ relaxation using localanaesthesia. Obstetrics and Gynaecology 86(6): 922-924.

2. Hill N, Gupta A, Zakaryan A, Morey R (2011) The success of 6-hour hospital discharge on patients having vaginal repair operation using a new conscious section technique. J Obstet Gynaecol 31(2): 149-151.

3. Kuhn A, Gelman W, O'Sullivan S, Monga A (2006) The feasibility, efficacy and functional outcome of local anaesthetic repair of anterior and posterior vaginal wall prolapse. Eur J Obstet Gynecol Reprod Biol 124(1): 88-92.

4. Dunhill M, Buchsbauon E, Duecy E (2005) Local anaesthesia with sedation for transvaginal correction of advanced genital prolapse. American Journal of Obstetrics and Gynaecology 193(6): 2173-2175.

5. Subramanya J, Curtiss N, Balachandran A, Puckett J (2016) Should we use a vaginal pack to reduce blood loss at the time of prolapse surgery? Eur J Obstet Gynecol Reprod Biol 206: 181-183.

6. Mitchell M (2015) Home recovery following day surgery: a patient perspective. J Clin Nurs 24(1-3): 415-427.

\section{Your next submission with Juniper Publishers} will reach you the below assets

- Quality Editorial service

- Swift Peer Review

- Reprints availability

- E-prints Service

- Manuscript Podcast for convenient understanding

- Global attainment for your research

- Manuscript accessibility in different formats ( Pdf, E-pub, Full Text, Audio)

- Unceasing customer service

Track the below URL for one-step submission https://juniperpublishers.com/online-submission.php 